\title{
Cell-Sensitive Phase Contrast Microscopy Imaging by Multiple Exposures
}

\author{
Zhaozheng Yin ${ }^{\mathrm{a}}$, Hang $\mathrm{Su}^{\mathrm{b}}$, Elmer $\mathrm{Ker}^{\mathrm{c}}$, Mingzhong $\mathrm{Li}^{\mathrm{a}}$, Haohan $\mathrm{Li}^{\mathrm{a}}$ \\ ${ }^{a}$ Missouri University of Science and Technology, USA \\ ${ }^{b}$ Tsinghua University, China \\ ${ }^{c}$ Stanford University, USA
}

\begin{abstract}
We propose a novel way of imaging live cells in a Petri dish by the phase contrast microscope. By taking multiple exposures of phase contrast microscopy images on the same cell dish, we estimate a cell-sensitive camera response function which responds to cells' irradiance signals but generates a constant on non-cell background signal. The result of this new microscopy imaging is visually superior quality, which reveals the appearance details of cells and suppresses background noise near zero. Using the cell-sensitive microscopy imaging, cells' original irradiance signals are restored from all exposures and the irradiance signals on non-cell background regions are restored as a uniform constant (i.e., the imaging system is sensitive to cells only but insensitive to non-cell background). The restored irradiance signals greatly facilitate the cell segmentation by simple thresholding. The experimental results validate that high quality cell segmentation can be achieved by our approach.
\end{abstract}

Keywords: Microscopy image analysis, microscopy imaging model, image restoration, cell image segmentation

\section{Introduction}

Phase contrast microscopy imaging (Zernike (1955)), a non-invasive technique, has been widely used to observe live cells without staining them (Kanade et al. (2011); Rittscher (2010)). A typical pipeline of phase contrast microscopy image formation is summarized in Fig.1, which consists of a phase contrast microscope and a digital camera to record time-lapse microscopy images on cells to analyze their properties. Starting from the beginning of the pipeline, the illuminance $(L)$ passes through a Petri dish 


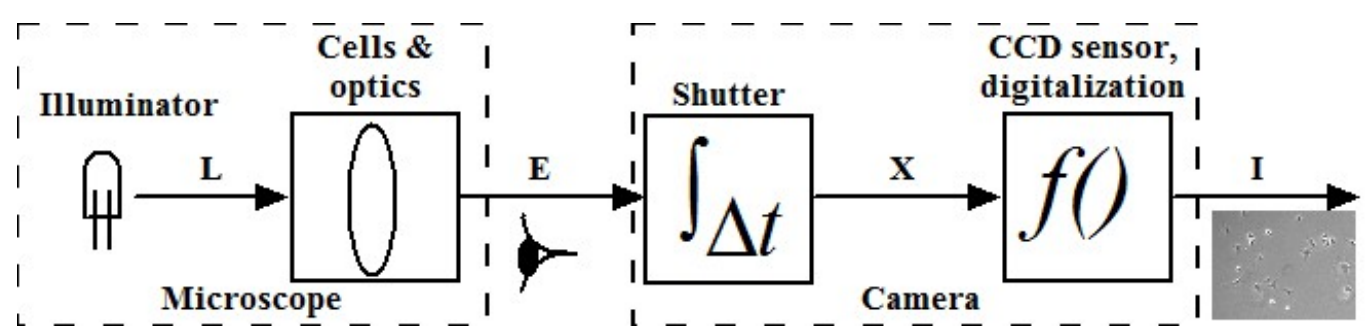

Figure 1: Phase contrast microscopy imaging consists of two components: the microscope optics and a digital camera. The illuminance $L$ from an illuminator passes through a Petri dish culturing cells and the optics of phase contrast microscope, forming an irradiance $E$ which is visible to human eyes and digital cameras. The camera records the irradiance $(E)$ within an exposure duration $(\Delta t)$ into an accumulated irradiance $(X, X=E \Delta t)$. Then, the irradiance $X$ is transformed into pixel values $(I)$ in digital images by a CD camera whose sensor response function is $f$, i.e., $I=f(E \Delta t)$.

culturing cells and phase contrast microscope optics, and generates the irradiance $(E)$ observable by naked human eyes or digital cameras. The camera captures the irradiance $(E)$ within an exposure duration $(\Delta t)$ and transforms the accumulated irradiance $(X, X=E \Delta t)$ into pixel values $(I)$ in digital images by a CCD camera whose sensor response function is $f$, i.e., the pixel value in a microscopy image is a function of irradiance and exposure time: $I=f(E \Delta t)$.

To automatically monitor cells' behavior by computational algorithms, cells need to be segmented and localized in images first, then they can be tracked and analyzed over time by object tracking algorithms such as data association (Bise et al. (2011)) and minimum-cost flow algorithm (Padfield et al. (2011)). Well-known image segmentation algorithms such as thresholding, morphological operations, watershed, level-set and Laplacian-of-Gaussian filtering, have been explored in various cell image analysis systems (House et al. (2009); Li et al. (2008); Otsu (1979); Smith et al. (2008); Wu et al. (1995); Yang et al. (2005)). Since these cell segmentation algorithms overlook the specific image formation process of phase contrast microscopy, recently the imaging model of phase contrast microscope optics and its related features have been exploited to facilitate the cell segmentation (Yin et al. (2012); Su et al. (2013)). However, the work on imaging model of phase contrast optics (Yin et al. (2012)) focuses on the front-end of the complete imaging pipeline (the microscope in Fig.1) but ignores the rear-end of the pipeline (the camera in Fig.1).

In fact, different camera settings also affect the cell image analysis. In Ali 
et al. (2007), a defocused image is used to initialize a level-set-based method to localize cell boundaries and then the contour sequentially evolves toward the correct boundaries with a series of variable focus images. In addition to the camera focal length, the exposure setting of a camera also plays a critical role in the cell image analysis. As shown in Fig.2, on the same cell dish, too short or too long exposure duration yields under-exposure (Fig.2(a)) or overexposure (Fig.2(d)), respectively. In the under-exposed image (Fig.2(a)), we can observe the halo artifacts and bright cells which are having mitotic or apoptotic events, but the other normal cells are with low contrast compared to the background. In the over-exposed image (Fig.2(d)), we can observe the normal cells, but the bright cells are over saturated and become hard to be seen.

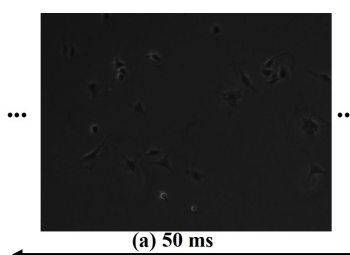

Under exposed

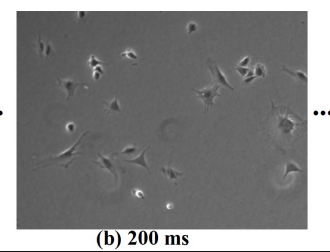

(b) $200 \mathrm{~ms}$

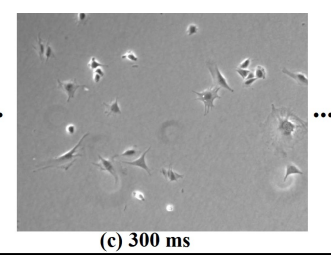

(c) $300 \mathrm{~ms}$

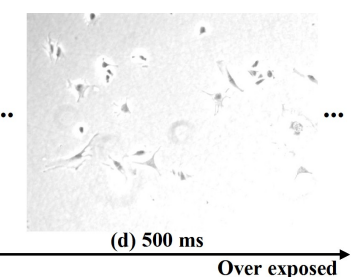

Over exposed

Figure 2: Multiple exposures on the same cell dish (ms: millisecond).

Based on the phase contrast microscopy imaging pipeline (Fig.1) and observation on differently exposed images (Fig.2), we are motivated to think of several intriguing problems in microscopy imaging and cell image analysis:

- Biologists and algorithm developers usually choose a fixed exposure setting based on their own preferences (e.g., Fig.2(b) or (c)) to observe cells and analyze their images. But, less attention is paid to choose an optimal exposure setting or explore multiple exposures for image analysis. Since a single fixed exposure might ignore some cells or overlook some cell details, can we combine multiple-exposed images to reveal all appearance details of all cells such that cell image analysis algorithms will not be affected by different exposures?

- Biologists and algorithm developers aim to analyze cells' original properties encoded in the irradiance signal E. However, a camera's sensor response function $f$ is usually nonlinear (Debevec and Malik (1997); Larson et al. (1997)), i.e., the acquired image signal $I$ is not linearly 
proportional to cells' irradiance $E$. Therefore, all cell image analysis algorithms directly based on image signal $I$ may deviate from the expected analysis on cells' original physical properties encoded in E. Can we restore the irradiance signal E from the image signal I for cell signal analysis?

- During the microscopy imaging, we hope to have high-quality images on cells only without any noise from the background of the culturing Petri dish. But, the pixels of non-cell regions in a dish may exhibit a significant amount of noise as shown in Fig.3, causing a nonuniform background and low contrast between cell and background pixels. Can we create a microscopy imaging system that is only sensitive to cells (i.e., insensitive to background, with a constant irradiance signal on non-cell background regions, facilitating the cell segmentation task)?
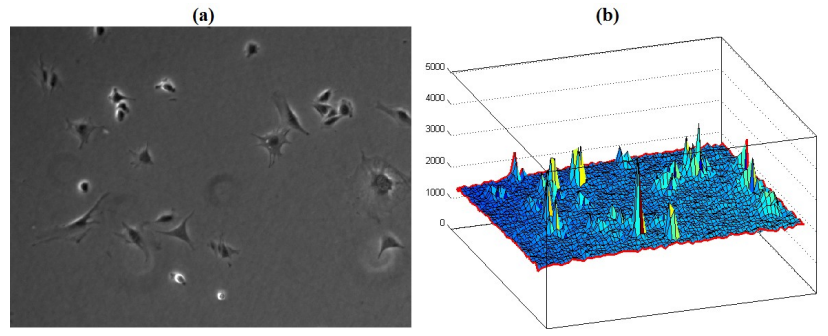

Figure 3: Noise from the background. (a) A phase contrast microscopy image; (b) The pixel values of (a) visualized in a surf view. Red curves around the surface boundary highlight the fluctuation of pixel values.

Based on the above motivations, we propose to create a cell-sensitive phase contrast microscopy imaging system by considering the rear-end of the imaging pipeline. First, multiple microscopy images with various exposure durations are taken on the same cell dish. Then, the camera response function is estimated from the set of multiple exposed images with a constraint that the irradiance signals of non-cell background pixels should be a constant, thus the imaging system is virtually sensitive to cells only. Finally, the irradiance signal map of cells is restored from all exposures based on the cell-sensitive camera response function. The restored irradiance signal map has a few advantages to echo our motivations:

- Each phase contrast microscopy image with a specific exposure only has a limited range of luminance levels, resulting in the loss of de- 
tails in bright or dark regions, but the restored irradiance signal map compensates for this loss of details by combining multiple small ranges of illuminance levels into a broader range, revealing more appearance details of cells;

- The restored signal map is directly related to the irradiance after the microscope optics ( $E$ in the imaging pipeline of Fig.1), thus removing the nonlinear function mapping introduced by the camera sensor and uncovering the true cell properties;

- In the restored irradiance signal map, non-cell background region has a constant irradiance different from those of cells, which will greatly facilitate the cell segmentation task since simple thresholding techniques can be applied to classify cell signals from background signals (i.e., the pixels whose irradiance signals differ from those of background pixels by a threshold are classified as cell pixels).

In the following of this paper, we describe the data acquisition in Section 2. Then, we introduce the details of cell-sensitive phase contrast microscopy imaging and cell segmentation in Section 3. The experimental results are discussed in Section 4, followed by a conclusion in Section 5.

\section{Data Acquisition}

The camera response function can be recovered by taking multiple images of a static scene with different exposures (Debevec and Malik (1997); Grossberg and Nayar (2003)). Since cells migrate slowly in a dish, phase contrast microscopy images are usually taken every 5 minutes to monitor the cells' proliferation process over weeks. As shown in Fig.4, the movement of cells between 5 minutes are not visually noticeable except some small fast-moving floating dirt in the dish which is considered as distracting noise during the cell-sensitive imaging. We will explain how to handle the distracting fastmoving foreground pixels in Section 3.4. In our microscopy imaging, we take multi-exposure images every 5 minutes with a range of known exposure durations $([50,100,200,300,400,500] \mathrm{ms}$, in total, about 1.55 seconds per set). The process to acquire such a set (1.55 seconds) is very fast compared to the time-lapse interval ( 5 minutes), hence we assume the irradiance signal for each pixel is stable when capturing such a set of multiple exposures and 
there is no need to do any image registration due to cells' slow motion. Common image acquisition software such as Axiovision 4.7 from Zeiss in our lab can routinely capture the multi-exposures every 5 minutes.

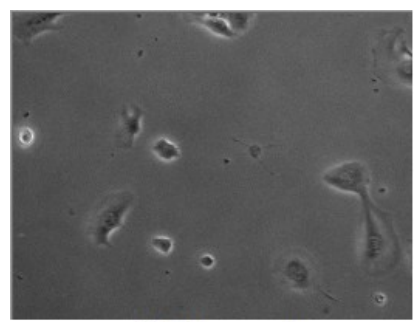

(a) Frame t

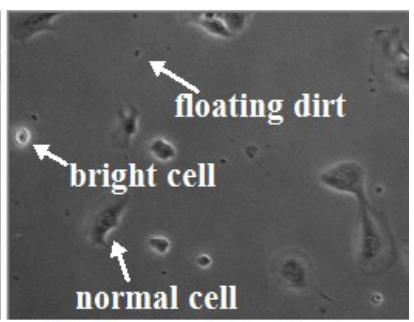

(b) Frame $\mathbf{t}+5$ minutes

Figure 4: Two phase contrast microscopy images captured at a 5-minute interval on the same Petri dish culturing $\mathrm{C} 2 \mathrm{C} 12$ cells used in stem cell research. The cells migrate slowly. The only objects moving swiftly in the images are some small floating dirt in the dish.

In Fig.4, there are two types of cells: normal cells and bright cells. The normal cells have dark appearance with halo artifacts surrounding them. The bright cells are mitotic (dividing) or apoptotic (dying) cells which have increased thickness/height compared to their surrounding medium. Cells that are in the process of mitosis or apoptosis are often spherical. This shape is due to a thermodynamic effect to minimize the contact between hydrophobic cell membrane molecules and the surrounding hydrophilic aqueous medium. As a result of this spherical shape, mitotic/apoptotic cells are often taller compared to well-attached and well-spread out normal cells.

During the phase contrast microscopy imaging, there is no negative impact on live cells when taking multiple images, because the amount of light energy cells are exposed to is very low. We have routinely exposed cells for 96 hours continuously with no photo toxic effects. However, invasive fluorescence imaging will have a detrimental effect on cells over the long term depending on the light source, filters and light intensity. Thus, phase contrast microscopy imaging with multi-exposures is suitable for long-term cell monitoring without invasive staining required by fluorescence imaging.

\section{Cell-Sensitive Phase Contrast Microscopy Imaging}

In this section, first we introduce how to estimate the cell-sensitive camera response function, based on which we then describe how to restore the 
irradiance signal with a uniform background. Then, we present how to perform easy cell segmentation on the restored irradiance signal map by simple thresholding. Finally, we introduce an iterative algorithm to automatically select pixel samples for estimating the camera response function.

\subsection{Estimate the Cell-Sensitive Camera Response Function}

Let $E_{i}$ be the irradiance at the $i_{t h}$ pixel location in a cell dish. $I_{i j}$, the intensity of the $i_{t h}$ pixel in the $j_{t h}$ image with exposure duration $\Delta t_{j}$, is computed by

$$
I_{i j}=f\left(E_{i} \Delta t_{j}\right),
$$

where $f$ is the camera response function. $f$ is assumed to be the same for all pixel locations on the camera sensor. Parametric models have been applied to represent the camera response function. For example, a gamma curve function is used as the response function in (Farid (2001)) and a polynomial function is used as the parametric representation of the camera response function in (Kuthirummal et al. (2008)). Rather than using a parametric model, 201 real-world camera response functions for common brands of CCDs as well as video and digital cameras have been estimated in (Grossberg and Nayar (2003)). All of the camera response functions in the database are monotonic, which are invertible. Without any exception, we assume our camera response function is also monotonic and invertible. Since a camera's response function may vary significantly from an analytic format, we do not assume any parametric model in our estimation of camera response function. Computing the inverse function of Eq.1 and taking the logarithm on both sides, we have

$$
\log f^{-1}\left(I_{i j}\right)=\log E_{i}+\log \Delta t_{j} .
$$

We formulate a constrained least square minimization problem to solve the unknown camera response function and irradiance signal 


$$
\begin{aligned}
O(g, E)= & \sum_{i=1}^{N} \sum_{j=1}^{P}\left\{\omega\left(I_{i j}\right)\left[g\left(I_{i j}\right)-\log E_{i}-\log \Delta t_{j}\right]\right\}^{2} \\
& +\alpha \sum_{i \in[1, N], i \in \Psi}\left(\log E_{i}\right)^{2} \\
& +\beta \sum_{I=I_{\min }+1}^{I_{\max }-1}\left[\omega(I) g^{\prime \prime}(I)\right]^{2},
\end{aligned}
$$

where $g=\log f^{-1}$. Note that, we only need to estimate a look-up table for the function $g$ since its input domain is the pixel value range. For a 12-bit TIFF image, the look-up table's length is 4096 with the minimum pixel value $I_{\min }=0$ and the maximum $I_{\max }=4095 . \quad N$ and $P$ denote the number of pixel samples and exposed images, respectively.

The first term in Eq.3 is the data-fitting cost. The second term in Eq.3 is the regularization cost which enforces the logarithm of background irradiance to zero (i.e., the background irradiance is driven to be a constant value, one). $\Psi$ is a set of some representative background pixel samples (how to automatically select these samples will be explained later in Section 3.4). The constant background will facilitate cell image analysis such as cell image segmentation by simple thresholding. In reality, the irradiance of background pixels may not be a constant due to natural variations. The goal of this constraint is to recover a camera response function similar to Fig.5. Given a wide range of background pixel intensities, their corresponding irradiance values are recovered into a small range around zero. This regularization is still applicable when there is a slow irradiance gradient across the image. If the value range of the background irradiance is different from the cell irradiance, we can still segment cells out of the background. In fact, the irradiance from the background is different from the irradiance from cells in the phase contrast microscope. Otherwise, we can not visualize cells through the ocular lens using human eyes.

The third term in Eq.3 is a smooth term avoiding overfit on the data, which is defined by the second order derivative:

$$
g^{\prime \prime}(I)=g(I-1)-2 g(I)+g(I+1) .
$$

The second derivative measures the discontinuity of the curve function. When the second derivative is high, the curve has a sharp curvature. Therefore, 


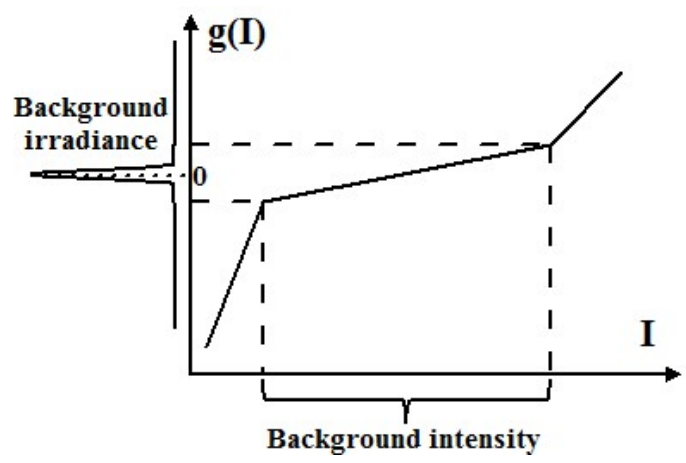

Figure 5: We are estimating a camera response function such that the logarithm of irradiance values $(g(I))$ of background pixels are recovered into a small range close to the zero, while the irradiance details of cell pixels are well recovered by the two ends of the camera response function.

minimizing the second derivative can ensure the smoothness during curve fitting (Wolberg and Alfy (2002)). A common camera response function has a steep slope at the end of the camera response curve due to over-saturation or under-exposure. In phase contrast microscopy images, the objects-in-interest (cells) are either dark or bright while the background pixels have a broad value range in the middle. To visualize the cells better, we can introduce a weight function $\omega(I)$ to emphasize the smoothness of the two ends of the camera response function. For example, a simple inverted hat function is a good choice for $\omega(I)$ :

$$
\omega(I)=\left|I-\frac{I_{\min }+I_{\max }}{2}\right| .
$$

As shown in Fig.6, the inverted hat function emphasizes more on the two ends of the camera response curve (i.e., sensitive to cells) and less on the middle (i.e., to have a flat background region).

$\alpha$ and $\beta$ in Eq.3 are coefficients to balance the three cost terms. In our experiments, we choose fixed coefficients with $\alpha=1000$ and $\beta=100$.

Since the objective function in Eq.3 is quadratic, taking its derivatives regarding to $g$ and $E$ and setting them to zero lead to an overdetermined system of linear equations $(\mathbf{A x}=\mathbf{b})$ which can be solved using the pseudo inverse or singular value decomposition method (Yin et al. (2014)). The unknown variable in the linear equation system is $\mathbf{x}=\left[g\left(I_{\min }\right), \ldots, g\left(I_{\max }\right), \log E_{1}, \ldots, \log E_{N}\right]$ whose first part $\left(\left[g\left(I_{\min }\right), \ldots, g\left(I_{\max }\right)\right]\right)$ is the cell-sensitive camera response function, a look-up table. Given $N$ pixels from $P$ multi-exposed images, we 


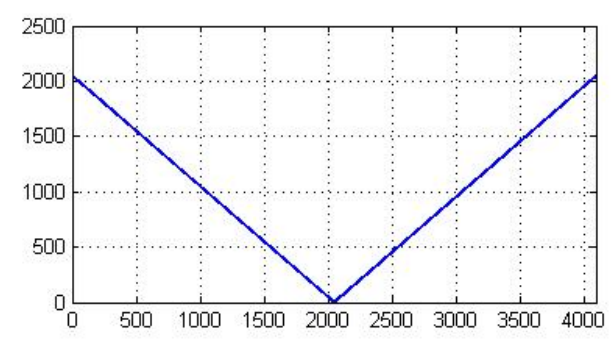

Figure 6: Weight function on the smoothness constraint.

have $\left(I_{\max }-I_{\min }+1\right)+N$ unknown variables in $\mathbf{x}$. To avoid rank deficiency in the linear equation system, we require the number of data fitting equations $N P>\left(I_{\max }-I_{\min }+1\right)+N$. For our experiments, $P=6, I_{\min }=0$ and $I_{\max }=4095$. We choose $N=1000$ to satisfy this requirement.

Fig.7(a) shows the estimated cell-sensitive camera response function (black curve) from a set of multiple exposed images. The samples from different exposed images (marked by circles with different colors in Fig.7(a)) cover different portions of the camera response curve. As shown in Fig.7(a), the inverse of our camera response function $\left(g=f^{-1}\right)$ is monotonic and invertible, therefore, $g$ is a one-to-one mapping from image intensities to irradiance values. Given a fixed camera and a fixed culturing dish, the response curve only needs to be estimated once using the first set of multiple exposed images and then it can be applied to successive sets of multi-exposures for time-lapse irradiance signal restoration. As shown in Fig.7(b), the cell-sensitive response curve is estimated every 1 hour using images from a 5-hour time-lapse cell image sequence captured on the same dish, and the six camera response curves overlap each other pretty well. Different cameras and culturing dishes with different backgrounds may have different cell-sensitive response curves, as shown in Fig.7(c) which includes three slightly different response curves on three different dishes.

\subsection{Restoring the Irradiance Signal}

After the cell-sensitive camera response function is estimated from the first set of multi-exposed images, for any other sets in the time-lapse image sequence, we can easily restore their relative irradiance signals from image pixel values by 


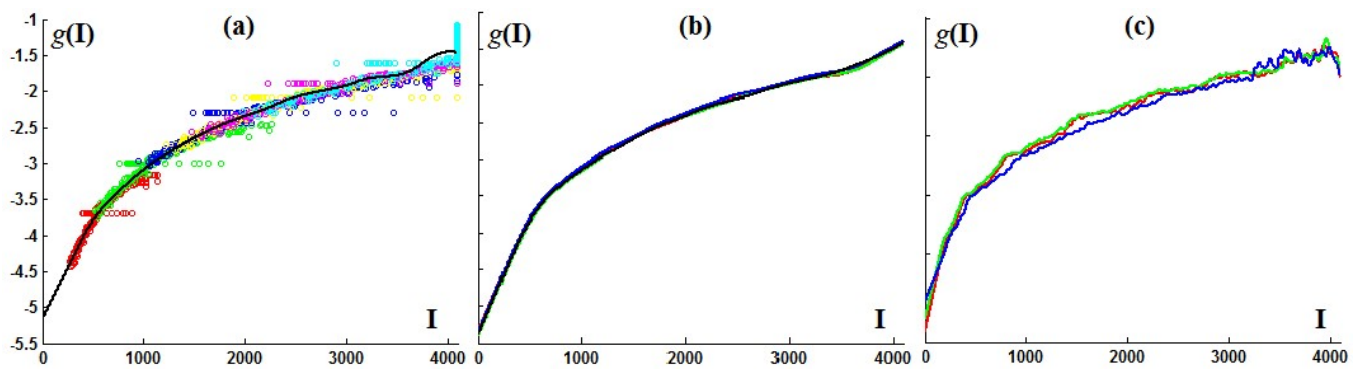

Figure 7: Camera response curves. (a) The camera response function (black curve) estimated from multi-exposures on the same cell dish. The circles shown with different colors represent samples from different exposed images. (b) The response function of a camera estimated from six sets of multi-exposures captured every 1 hour on the same cell dish. (c) The camera response function estimated from three different dishes.

$$
\log E_{i}=\frac{\sum_{j=1}^{P} w\left(I_{i j}\right)\left(g\left(I_{i j}\right)-\Delta t_{j}\right)}{\sum_{j=1}^{P} w\left(I_{i j}\right)},
$$

where all the exposures are used to restore the irradiance signal robustly.

\subsection{Cell Image Segmentation}

Fig.8(a) shows one of a set of multi-exposed phase contrast microscopy images. Fig.8(b) is the restored irradiance signal map whose surf view (Fig.8(c)) clearly demonstrates that the non-cell background region has uniform background and the contrast between cells and background in the restored irradiance signal map is high. We sort the irradiance values in an ascent order (Fig.8(d)). The majority of the 1.4 million pixels of a 1040x1388 signal map has $E=1$ (i.e., $\log E=0$, which is the goal of the second regularization term in Eq.3).

In phase contrast microscopy imaging, there are mainly three types of pixels: background pixels; bright pixels (e.g., mitotic/apoptotic cells or halos around normal cells); and dark pixels (e.g., migrating normal cells). Background pixels are commonly brighter than normal cell pixels but darker than bright pixels, as shown in Fig.8(a) and (b). The three types of pixel patterns are also reflected in Fig.8(d) where the majority flat region of the curve belongs to background pixels while the low and high ends of the curve are contributed by the dark and bright pixels, respectively.

Based on the restored irradiance signal map, a simple thresholding-based segmentation method is utilized. The value distribution of the restored ir- 
(a)

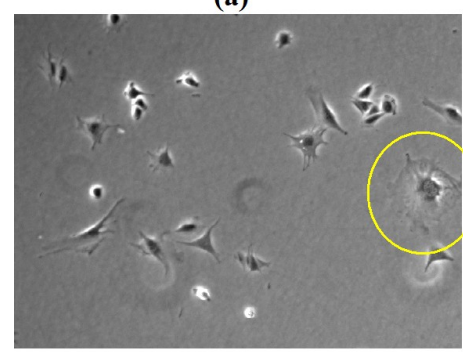

(d)

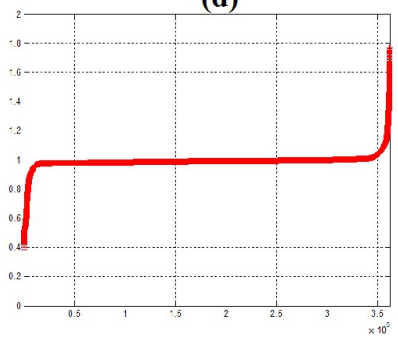

(g) (b)

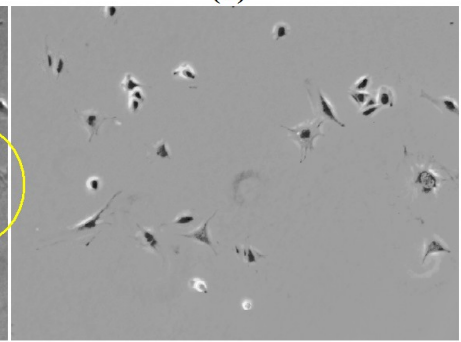

(e)

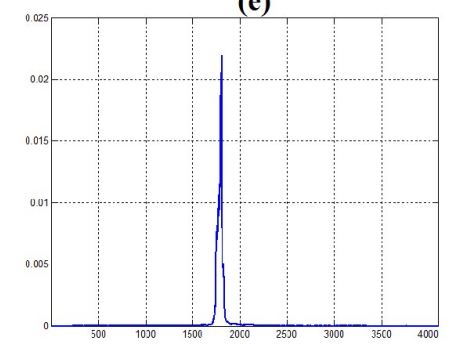

(h)

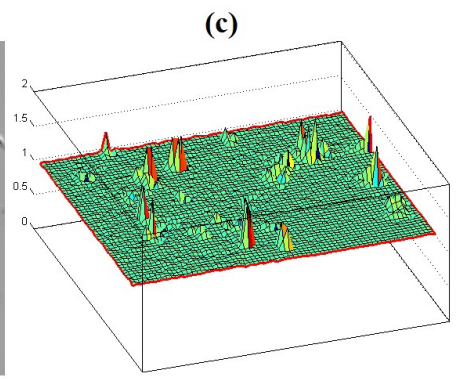

(f)

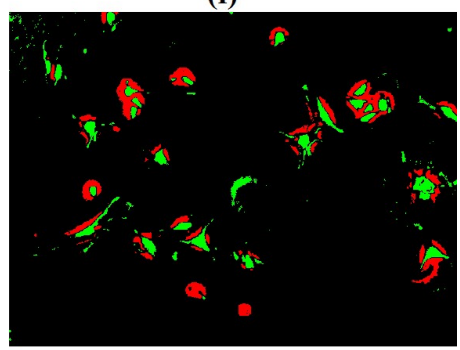

(i)
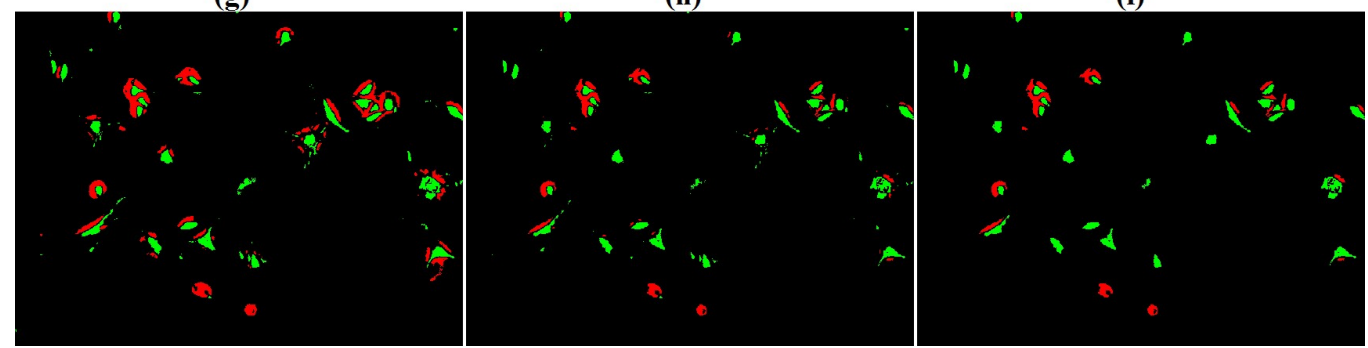

Figure 8: Cell segmentation. (a) One of a set of multi-exposed phase contrast microscopy images; (b) Restored irradiance signal map with constant irradiance values on the background and high signal contrast; (c) Irradiance map shown in the surf view; (d) The sorted irradiance signal values; (e) The value distribution of the restored irradiance signal map; $(\mathrm{f}, \mathrm{g}, \mathrm{h})$ Segmentation by thresholding the irradiance signal using thresholds $\mu-k \sigma$ and $\mu+k \sigma$ where $k=1,2,3$, respectively. (green: dark cell pixels; red: bright cell pixels or halo pixels; black: background); (i) Segmentation results with isolated cell pixels removed. 
radiance signal map (Fig.8(e)) is modeled as a single Gaussian distribution with mean $(\mu)$ and standard deviation $(\sigma)$. Two thresholds are sufficient to segment dark/bright pixels out of the restored irradiance signal: any $E$ signal larger than $\mu+k \sigma$ is a bright pixel and any $E$ signal lower than $\mu-k \sigma$ is a dark pixel. Fig.8(f,g,h) show the segmentation results of Fig.8(a) when $k=1,2,3$, respectively. When $k=3$, the underlying assumption is $99 \%$ of the image pixels are background pixels with intensities in the range of $[\mu-3 \sigma, \mu+3 \sigma]$. In this paper, we use $\mu-3 \sigma$ and $\mu+3 \sigma$ as the two thresholds to separate normal cells, background and bright cells. Other methods to select the best thresholds can be the cross-validation in the training datasets (Yin et al. (2012)). After the pixel-wise thresholding (Fig.8(h)), the isolated pixels can be removed with the remaining big blobs representing cells (Fig.8(i)).

Note that, our proposed method flattens the background image and emphasizes the dark and bright pixels in the microscopy image, which may lead to over-segmentation (one cell is segmented into multiple segments) or missegmentation (part of the cell is not segmented), such as the cell in the yellow circle of Fig.8(a). It is very difficult to segment those cells whose peripheries are widely spread out with their intensities close to the background. We will explore this challenging segmentation in our future research. Thus, to obtain the ground-truth in the experiment evaluation of this paper, the annotators will only annotate the dark and bright cell regions without the wide-spread, low-contrast cell peripheries.

\subsection{Select Sample Pixels for Estimating the Camera Response Function}

When estimating the camera response function in Eq.3, we need $N$ pixel samples. Note that, there is no need to select all image pixels for the estimation. A small set of representatives will be enough to estimate the cell-sensitive camera response function. To avoid biased estimations, we uniformly divide the image coordinate into 100 rectangular regions (a 10x10 grid). Within each region, we randomly select $N / 100$ pixels whose values are evenly distributed from the minimum to the maximum in this region. In total, we obtain $N$ sample pixels, some of which will be on the background. Identifying background pixels out of the $N$ sample pixels is required by the regularization term in Eq.3 (pixel set $\Psi$ ). We propose a recursive approach to identify background pixel samples and estimate the camera response function.

First, an initial set of background pixels are identified by using the overand under-exposed images. As shown in Fig.9(a), many background pixels 

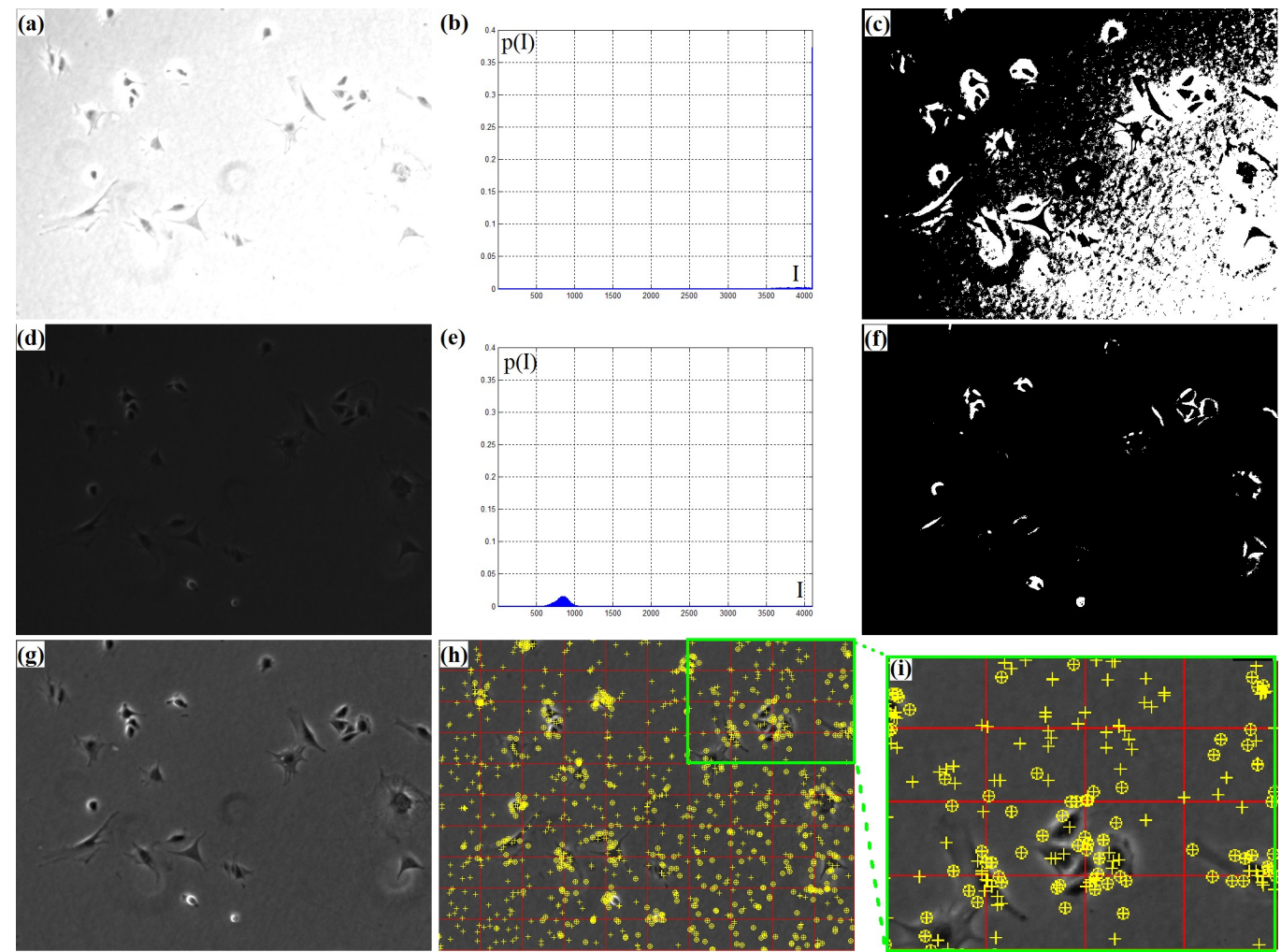

Figure 9: Initial selection of pixel samples and identification of background samples. (a) An over-exposed image; (b) The impulse distribution of pixel intensities of (a); (c) The bitmask of saturated pixels; (d) An under-exposed image; (e) The Gaussian distribution of pixel intensities of (d); (f) The bitmask of the bright and background pixels; (g) An image with 300ms exposure duration; (h) Pixel samples uniformly selected from spatial regions and value ranges; (i) Many miss-identifications of background samples ('+' and 'o' denote the selected pixel samples and initially identified background pixels, respectively).

and bright cell pixels are saturated in the over-exposed image, and its pixel intensity distribution has an impulse distribution around the maximum intensity (Fig.9(b)). Finding all the saturated pixels gives us the bitmask $M_{o}$ (Fig.9(c)). In the under-exposed image (Fig.9(d)), cells and background are dark with low contrast, and its intensity has a Gaussian distribution (Fig.9(e)). Using the threshold of $\mu+3 \sigma$ of this Gaussian distribution, we get the bitmask $M_{u}$ representing the bright cells and halo regions (Fig.9(f)). Therefore, those of the $N$ sample pixels which are from the mask $M_{o}$ but not from mask $M_{u}$ are considered as initial identification of background pixel samples. Fig.9(h) shows the selected pixel samples of the image in Fig.9(g), 
and Fig.9(i) is a zoom-in subimage where marker ' + ' denotes the selected pixel samples and marker 'o' denotes the initially identified background pixels. As we observe from Fig.9(i), the initial identification of background samples by simply thresholding over- and under-exposed images has many miss-identifications.

After obtaining the pixels samples, we estimate the cell-sensitive camera response function by solving Eq.3, restore the irradiance signal by Eq.6, segment the image into bright pixels, dark pixels and background by thresholding. Based on the segmented background regions, the background pixels in the $N$ samples are re-identified and the process is iterated until no change on the estimated camera response function. The process is summarized in the following Algorithm 1.

\section{Algorithm 1: Estimating the Cell-Sensitive Camera Response Func- tion}

Input: a set of multi-exposed images and the number of pixel samples $N$;

Output: the cell-sensitive camera response function $g$;

Initialization: select $N$ sample pixels and identify background samples of the $N$ pixels by thresholding the over- and under-exposed images; $i \leftarrow 0$, $g_{0}=\mathbf{0}$

\section{Repeat}

1: $i \leftarrow i+1$;

2: Estimate cell-sensitive camera response function $g_{i}$ using the $N$ sample pixels by solving Eq.3;

3: Restore the irradiance signal by solving Eq.6;

4: Segment the irradiance map into bright, dark and background pixels by thresholding;

5: Identify the background pixels in the $N$ samples;

6: $\Delta g=\left\|g_{i}-g_{i-1}\right\|$;

Until $\Delta g \leq \epsilon$.

Fig.10 shows several iterations of the recursive estimation. Fig.10(a) shows the sampled $N$ pixels. The initial sample selection has many missidentifications on background pixels, but the $N$ sampled pixels are gradually classified correctly in the following iterations. Fig.10(b) and (c) show the sorted signals and their signal value distributions, respectively. The initial image has a wide variance on the intensity values, but the restored irradiance signal has narrower Gaussian distributions. Fig.10(d) shows an original 
microscopy image and two sequentially restored irradiance signal maps. We can observe that the noise from the background is gradually reduced while the details of cells are well discovered. Fig.10(e) shows the cell segmentation results by thresholding based on the Gaussian distributions of the signals. The restored irradiance signal map greatly facilitates the cell segmentation.

The cell-sensitive camera response function $g$ is a look-up table (e.g., for a 12 bit image, its input domain is of 4096 values). We use the change of $g$ between two successive iterations, $\Delta g=\left\|g_{i}-g_{i-1}\right\|$, as the indicator of the algorithm convergence. As shown in Fig.11, the Algorithm 1 applied on the set of multi-exposed images corresponding to Fig.10 quickly converges in the first a few iterations.

The fast-moving floating dirt during the multiple exposures (Fig.4) causes irradiance fluctuation at those pixel locations when the floating dirt passes by. If the majority of pixel samples in the estimation of our camera response function (Eq.3) are from floating dirt pixels, forcing the intensities of floating dirt pixels to be a constant as other background pixels will estimate a wrong camera response function. However, in our biological experiments, the number of floating dirt pixels in the image is very rare compared to the total number of image pixels ( 1.4 million for an image with $1040 \times 1388$ pixels), as shown in Fig.4. When initially selecting $N(N=1000)$ pixels for the estimation of our camera response function based on the over- and under-exposed images (Fig.9), the probability to select the rare floating dirt pixels into the $N$ pixels is very low. Even some floating dirt pixels are mistakenly selected into the sample pool to estimate the camera response function in the first step, they will not skew the estimation of camera response function significantly since they are very rare in the $N$ pixel samples. Furthermore, the negative effect of initial selection of floating dirt pixels is mitigated promptly by our iterative algorithm (Algorithm 1) since the background pixel sample set $\Psi$ used in the estimation of camera response function is gradually purified during the iterations.

\section{Experimental Results}

\subsection{Qualitative Evaluation on Cell-Sensitive Imaging}

Human eyes can perceive a very high range of brightness values with a contrast ratio as high as $10^{6}: 1$, but a digital image has a much lower value range such as $[0,255]$ for an 8-bit JPEG image or [0,4096] for a 10-bit TIFF

image. Therefore, the observed irradiance $(E)$ by human eyes through a 


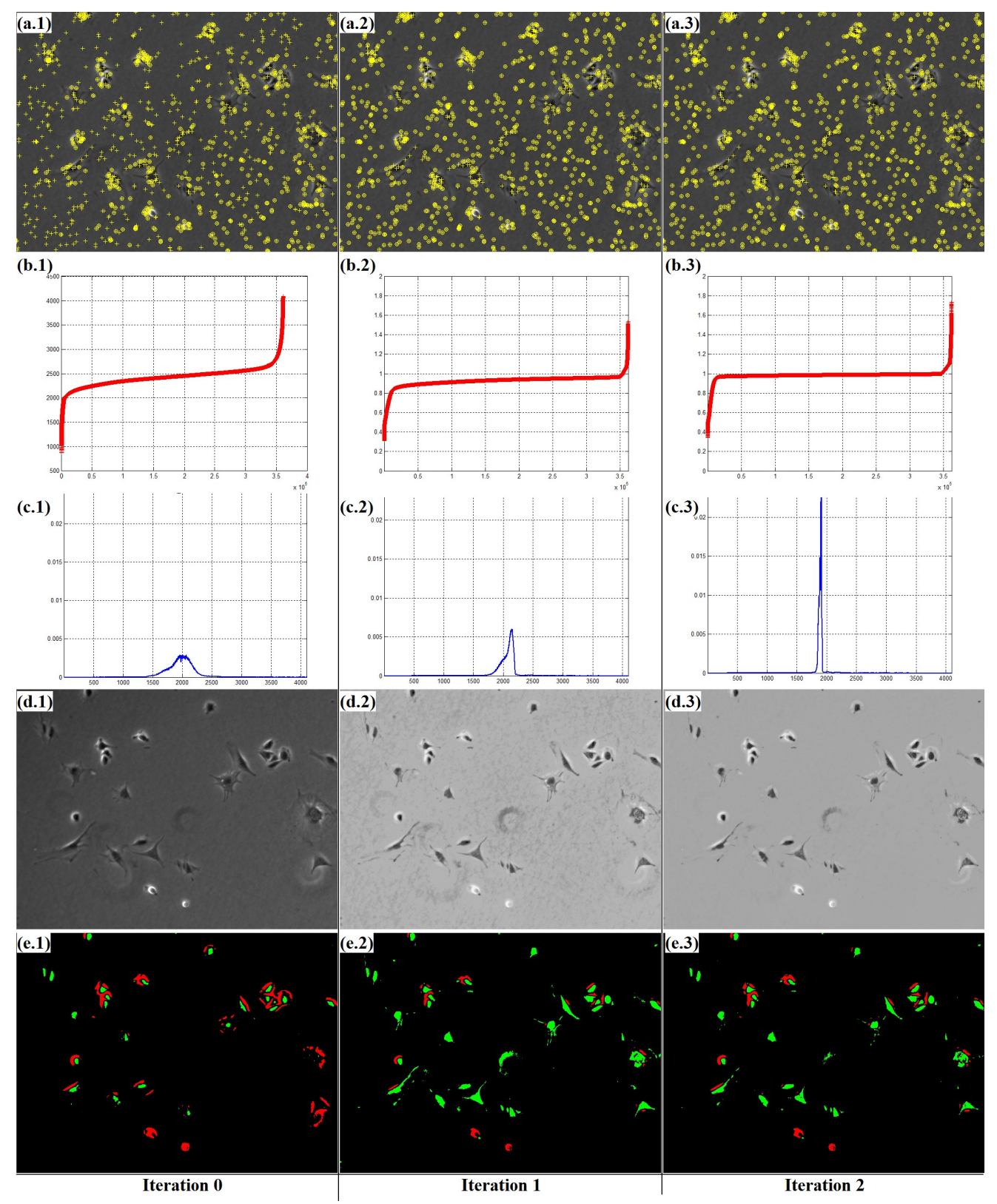

Figure 10: Iteratively estimating the cell-sensitive camera response function. (a) $N$ pixel samples; (b) Sorted signal values; (c) Signal value distributions; (d.1) One microscopy image; (d.2-d.3): Restored irradiance signal maps; (e) Image segmentation by thresholding (red, green and black represent bright, dark and background pixels, respectively). The first column shows the original input and the second and third columns show the first two iterations of applying Algorithm 1 on the input microscopy images. 


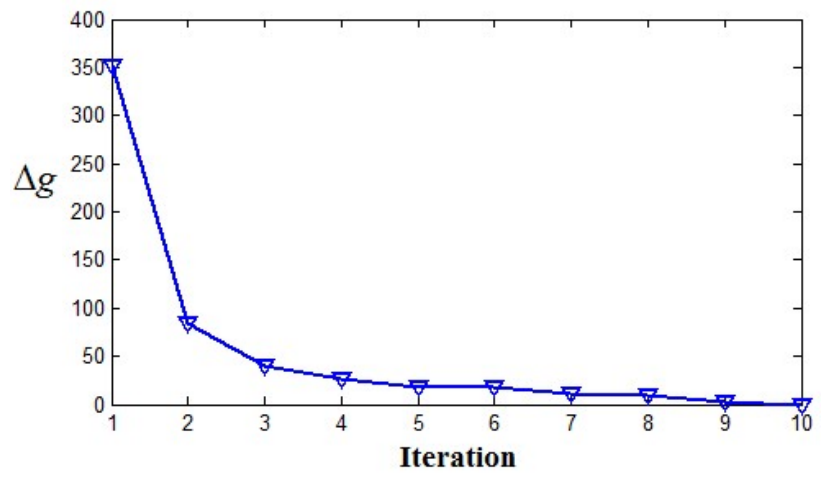

Figure 11: The convergence of iteratively estimating the cell-sensitive phase contrast microscopy imaging.
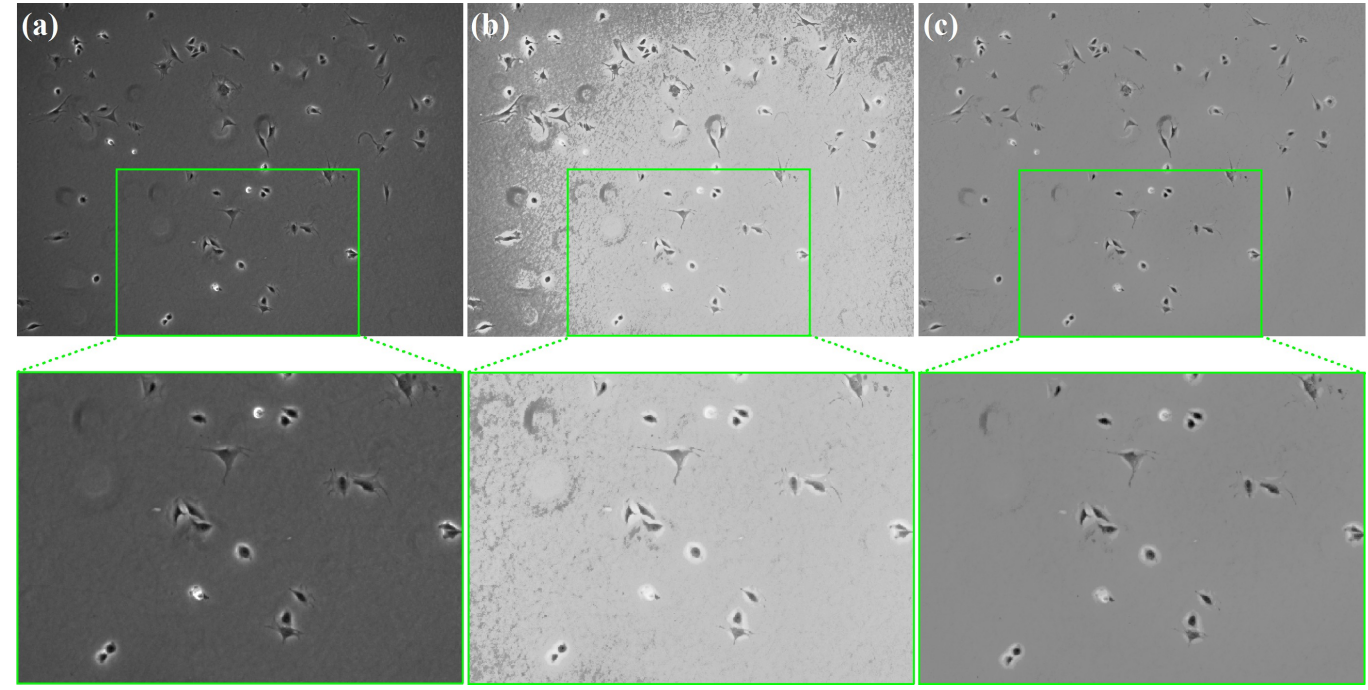

Figure 12: Comparison with high dynamic range (HDR) image. (a) A phase contrast image captured with exposure duration 200ms. (b) The HDR image created by multiexposures. (c) Our cell-sensitive imaging where the background is uniform and the image contrast between cells and the background is high. 
microscope's ocular lens may be different from the digital image observation (I). Multi-exposures have been used in Computer Graphics to create High Dynamic Range (HDR) images (Debevec and Malik (1997); Larson et al. (1997)). Fig.12(b) shows the HDR image created by using the code from Debevec and Malik (1997). Compared to the image with 200ms exposure duration (Fig.12(a)), the HDR image shows more image details but also amplifies the background noise largely. However, our cell-sensitive imaging is only sensitive to cells and enforces the background to have a constant irradiance, showing high contrast between cells and the uniform background, as shown in Fig.12(c). The novel cell-sensitive microscopy imaging recovers the observation at the end of ocular lens for better visualization and analysis on cells.

\subsection{Qualitative Evaluation on Cell Image Segmentation}

Fig.13 shows the qualitative comparison between our segmentation based on cell-sensitive imaging and two other related methods: (1) the restoration method based on phase contrast optics, which considers the front-end of the imaging pipeline (Yin et al. (2012)) and (2) multi-level Otsu thresholding method (Otsu (1979)). Compared to the original microscopy images (Fig.13(a)), our restored irradiance signal map (Fig.13(b)) has uniform background and high contrast between cells and background regions. Thresholding the irradiance signals can classify both bright and dark pixels from the background (Fig.13(c)). The other two methods are ran on the image with exposure duration 200ms. The phase contrast optics based method can locate the darkest nuclei regions but the low contrast cells and bright cells are not detected (Fig.13(d)). Considering there are three types of pixels in an image (bright, dark and background pixels), we use two-level Otsu thresholding to segment the image. Unfortunately, the multi-level Otsu thresholding does not perform well due to the non-uniform background (Fig.13(e)).

\subsection{Quantitative Evaluation on Cell Image Segmentation}

First, we collected multiple exposed microscopy images on three different cell dishes with low and high cell densities, and each set has 6 different exposure durations ([50 100200300400 500]ms). Two annotators manually labeled cell masks (both mitosis/apoptosis and normal cells but without halos) in all microscopy images with exposure duration $200 \mathrm{~ms}$. To reduce the inter-person variability, the intersection of their annotations is used as the ground truth. The segmentation accuracy (ACC) is defined as 


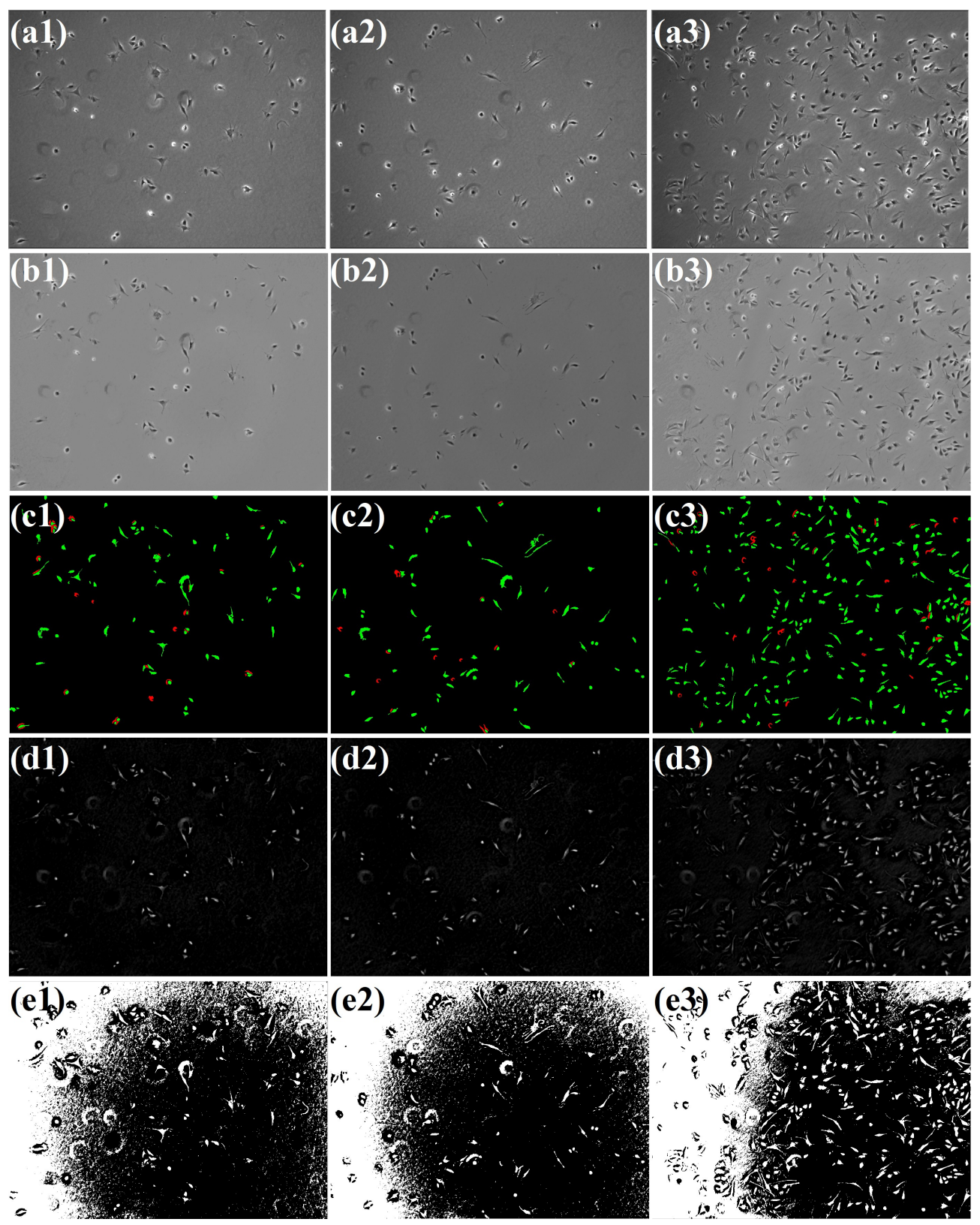

Figure 13: Three methods on one dish sequence. (a) Microscopy images with $200 \mathrm{~ms}$ exposure duration. (b) Restored irradiance signal by our cell-sensitive imaging. (c) The segmentation by thresholding the restored irradiance (red: mitosis/apoptosis cells or halos; green: normal migration cells). (d-e): Segmentation on the image with 200ms exposure duration by the method based on phase contrast optics model (Yin et al. (2012)) and Otsu thresholding (Otsu (1979)), respectively. 
$A C C=\left(|T P|+\left|N_{s}\right|-|F P|\right) /\left(\left|N_{s}\right|+\left|P_{s}\right|\right)$ where cell and background pixels are defined as positive samples $\left(P_{s}\right)$ and negative samples $\left(N_{s}\right)$, respectively. True positive $(T P)$ stands for cell pixels segmented by our method correctly and false positive $(F P)$ denotes cell pixels segmented by our method mistakenly. Table 1 compares the performance of three segmentation methods on three cell sequences in which our segmentation by cell-sensitive imaging achieves very high accuracy. We did not classify bright halos from bright mitosis/apoptosis cells in this paper, which lowers our cell segmentation performance a bit. The scope of this paper focuses on the cell sensitive microscopy imaging. In the future work, we will explore cell classification and tracking based on the cell-sensitive imaging.

\begin{tabular}{|c|c|c|c|}
\hline & Dish 1 & Dish 2 & Dish 3 \\
\hline Our cell-sensitive imaging & 0.993 & 0.994 & 0.975 \\
Optics model based method (Yin et al. (2012)) & 0.974 & 0.974 & 0.956 \\
Otsu threshold (Otsu (1979)) & 0.677 & 0.665 & 0.628 \\
\hline
\end{tabular}

Table 1: Cell segmentation accuracy of three methods on three dishes.

Since the cell segmentation ground-truth provided by human annotation has inter-person variability and intra-person variability (the same person may provide different annotations on the same image on different days), we also explored the fluorescence imaging for ground truth by staining the cells. Note that, the invasive staining is only applied for the purpose of obtaining ground-truth to evaluate our algorithm. In real experiments of monitoring live cells, we do not need to stain cells. We collect 10 sets of multi-exposed phase contrast microscopy images with their corresponding fluorescence images. Fig.14(a) and (d) show one example of the phase contrast image and its fluorescence image. The restored irradiance signal map based on our cellsensitive imaging is shown in Fig.14(b) where the cell details are well restored and the background is flattened. Fig.14(c) shows the image restoration results based on the phase contrast optics model (Yin et al. (2012)). Since the pixel values in the fluorescence image are continuous in the range of $[0,1]$, we need to threshold it to have the binary mask to perform the quantitative comparison on the cell segmentation. Different thresholds on the fluorescence image give us different ground-truth, as shown in Fig.14(e,f). We threshold our irradiance map (Fig.14(b)) and restoration results (Fig.14(c)) into binary masks, and compare them with ground-truth binary masks generated by thresholding the fluorescence image with different thresholds. As shown 
in Fig.15, both two methods achieve good performance on the pixel-wise cell segmentation, and our cell-sensitive imaging is slightly better than the image restoration method.
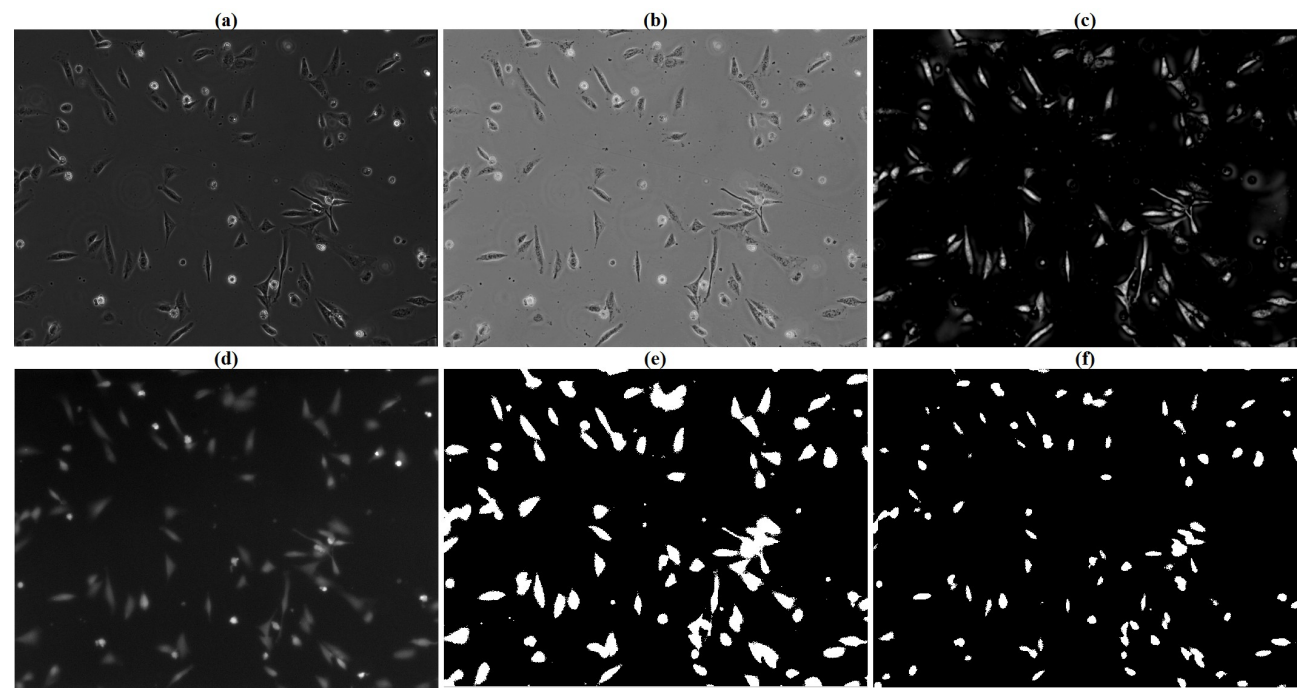

Figure 14: Phase contrast microscopy images with fluorescence images as ground-truth. (a) A phase contrast microscopy image with 200ms exposure duration. (b) Restored irradiance signal by our cell-sensitive imaging. (c) The image restoration result by the phase contrast optics model method (Yin et al. (2012)). (d) Fluorescence image on the same cell dish (pixel value $\in[0,1])$. (e,f) Segmenting the fluorescence image with threshold equaling to 0.1 and 0.2 , respectively.

\section{Conclusion}

We propose a highly promising way of imaging live cells in a Petri dish by the phase contrast microscope. A set of variously exposed phase contrast microscopy images on the same cell dish are used to estimate a cellsensitive camera response function, based on which cells' original irradiance signals are restored from all exposures while the irradiance signals on noncell background regions are restored as a uniform constant. Therefore, the imaging system is sensitive to cells but insensitive to non-cell background, which greatly facilitates the cell segmentation by simple thresholding. The experimental results show our approach can achieve high cell segmentation accuracy. Meanwhile, the result of cell-sensitive microscopy imaging is visually superior quality which reveals appearance details of cells from multiple exposures and suppresses the background noise. 


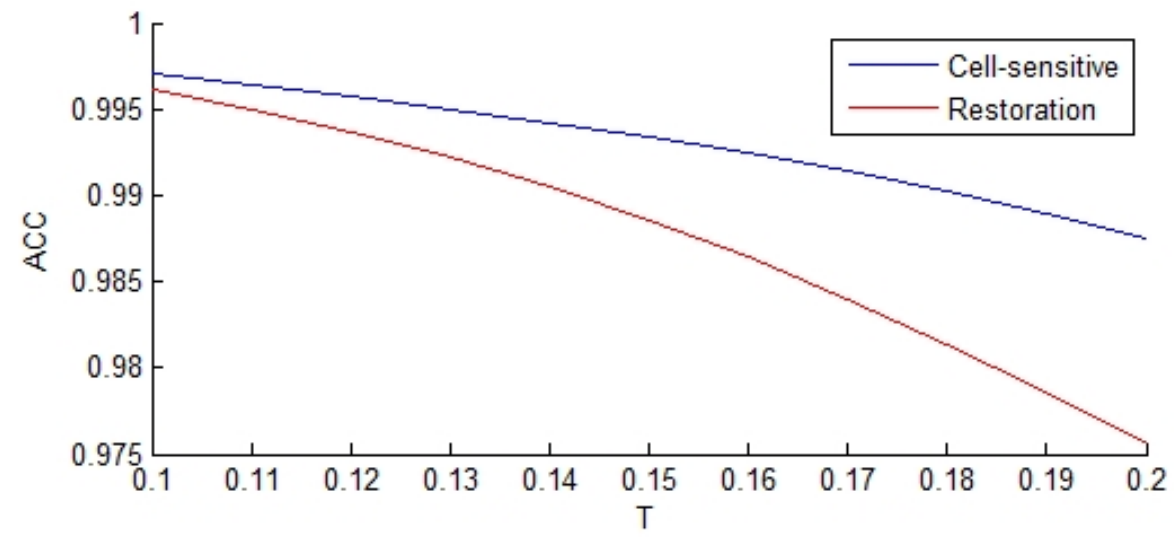

Figure 15: Cell segmentation by our cell-sensitive method is compared with the image restoration result (Yin et al. (2012)) in terms of accuracy (ACC), when different thresholds $(T)$ are applied to the fluorescence image to generate the ground-truth segmentation mask.

\section{Acknowledgement}

This research was supported by University of Missouri Research Board, Intelligent System Center and Center for Biomedical Science and Engineering at Missouri University of Science and Technology, National Science Foundation (NSF) EPSCoR grant IIA-1355406 and NSF CAREER award IIS1351049. Any opinions, findings, and conclusions or recommendations expressed in this material are those of the authors and do not necessarily reflect the views of NSF.

\section{References}

Rehan Ali, Mark Gooding, Martin Christlieb, and Michael Brady, "Phasebased Segmentation of Cells with Brightfield Microscopy," IEEE Symposium on Biomedical Imaging (ISBI), 2007.

Ryoma Bise, Zhaozheng Yin, and Takeo Kanade, "Reliable Cell Tracking by Global Data Association," IEEE International Symposium on Biomedical Imaging (ISBI), 2011.

Paul Debevec and Jitendra Malik, "Recovering High Dynamic Range Radiance Maps from Photographs," SIGGRAPH, 1997.

Hany Farid, "Blind Inverse Gamma Correction," IEEE Transactions on Image Processing, 10(10): 1428-1433, 2001. 
Michael Grossberg and Shree Nayar, "What is the Space of Camera Response Functions?," IEEE Conference on Computer Vision and Pattern Recognition (CVPR), vol.II, pp.602-609, 2003.

David House, Matthew L. Walker, Zheng Wu, Joyce Y. Wong, and Margrit Betke, "Tracking of Cell Populations to Understand their Spatio-Temporal Behavior in Response to Physical stimuli, "Workshop on Mathematical Methods in Biomedical Image Analysis (MMBIA), 2009.

Takeo Kanade, Zhaozheng Yin, Ryoma Bise, Seungil Huh, Sungeun Eom, Michael Sandbothe, and Mei Chen, "Cell Image Analysis: Algorithms, System and Applications," IEEE Workshop on Applications of Computer Vision (WACV), 2011.

Sujit Kuthirummal, Aseem Agarwala, Dan Goldman and Shree Nayar, "Priors for Large Photo Collections and What They Reveal about Cameras," European Conference on Computer Vision, pp.74-87, Oct, 2008.

Gregory Ward Larson, Holly Rushmeier, and Christine Piatko, "A Visibility Matching Tone Reproduction Operator for High Dynamic Range Scenes," IEEE Transaction on Visualization and Computer Graphics, 3(4): 291-306, 1997.

Kang Li, Eric Miller, Mei Chen, Takeo Kanade, Lee E. Weiss, and Phil G. Campbell, "Cell Population Tracking and Lineage Construction with Spatiotemporal Context," Medical Image Analysis, 12(1): 546-566, 2008.

Douglas B. Murphy, "Fundamentals of Light Microscopy and Electronic Imaging," Wiley, 2001.

Nobuyuki Otsu, "A threshold selection method from gray-level histograms" IEEE Transactions on Systems, Man, and Cybernetics, 9(1): 62-66, 1979.

Dirk Padfield, Jens Rittscher and Badrinath Roysam, "Coupled MinimumCost Flow Cell Tracking for High-throughput Quantitative Analysis," Medical Image Analysis, 15(4): 650-668, 2011.

Jens Rittscher, "Characterization of Biological Processes through Automated Image Analysis," Annual Review of Biomedical Engineering, 12: 315-344, 2010 . 
Kevin Smith, Alan Carleton, and Vincent Lepetit, "General Constraints for Batch Multiple-Target Tracking Applied to Large-Scale Video Microscopy," IEEE Conference on Computer Vision and Pattern Recognition (CVPR), 2008.

Hang Su, Zhaozheng Yin, Seungil Huh, and Takeo Kanade, "Cell Segmentation in Phase Contrast Microscopy Images via Semi-supervised Classification over Optics-related Features," Medical Image Analysis, 17(7): 746-765, 2013.

George Wolberg, and Itzik Alfy, "An Energy Minimization Framework for Monotonic Cubic Spline Interpolation," Journal of Computational and Applied Mathematics, 143(2): 145-188. 2002

Kenong Wu, David Gauthier, and Martin D. Levin, "Live Cell Image Segmentation," IEEE Transaction on Biomedical Engineering, 42(1): 1-12, 1995.

Fuxing Yang, Michael A. Mackey, Fiorenza Ianzini, Greg Gallardo, and Milan Sonka, "Cell Segmentation, Tracking, and Mitosis Detection Using Temporal Context," International Conference on Medical Image Computing and Computer Assisted Intervention (MICCAI), 2005.

Zhaozheng Yin, Mei Chen and Takeo Kanade, "Understanding the Phase Contrast Optics to Restore Artifact-free Microscopy Images for Segmentation," Medical Image Analysis, 16(5): 1047-1062, 2012.

Zhaozheng Yin, Hang Su, Elmer Ker, Mingzhong Li, and Haohan Li, "CellSensitive Microscopy Imaging for Cell Image Segmentation," International Conference on Medical Image Computing and Computer Assisted Intervention (MICCAI), 2014.

Frits Zernike, "How I discovered phase contrast," Science, 121: p345-p349, 1955. 
The pipeline of microscopy imaging

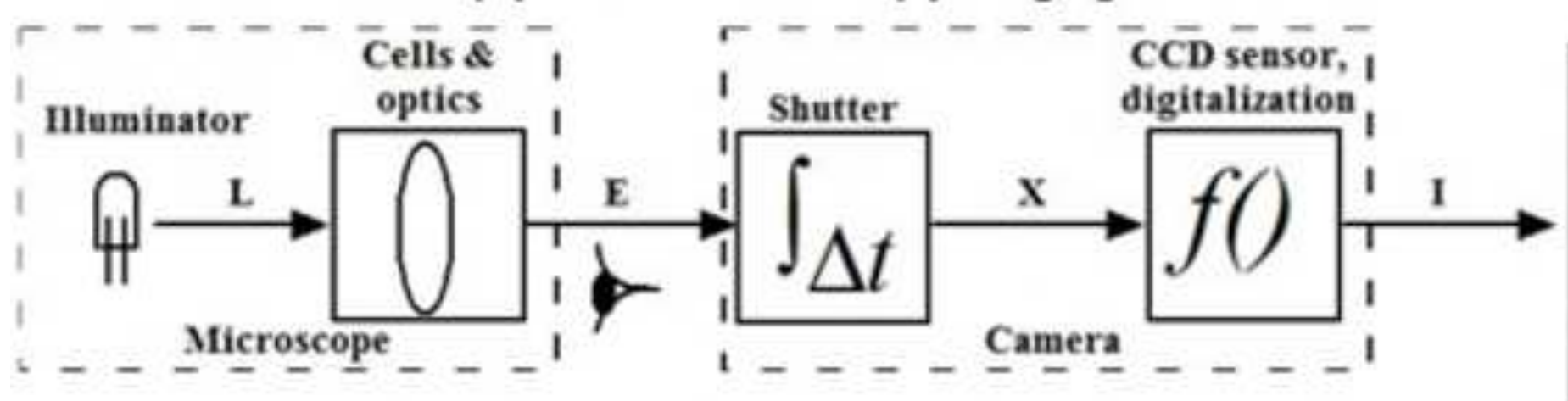

Cell-sensitive phase contrast microscopy imaging

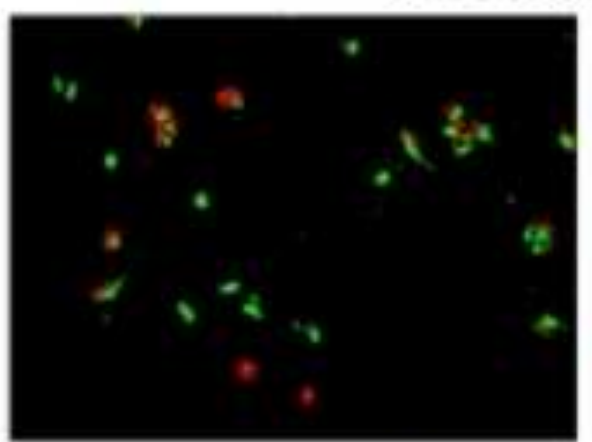

Fadilitate cell segmentation

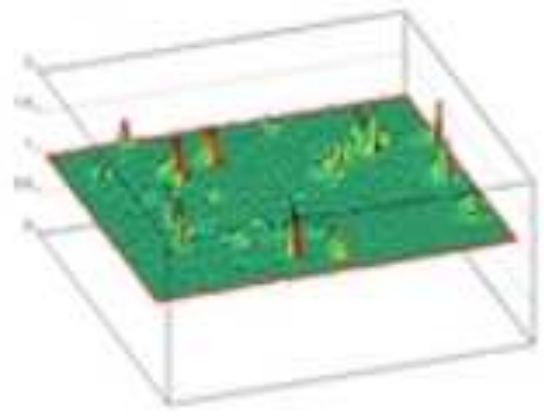

Suppress background noise

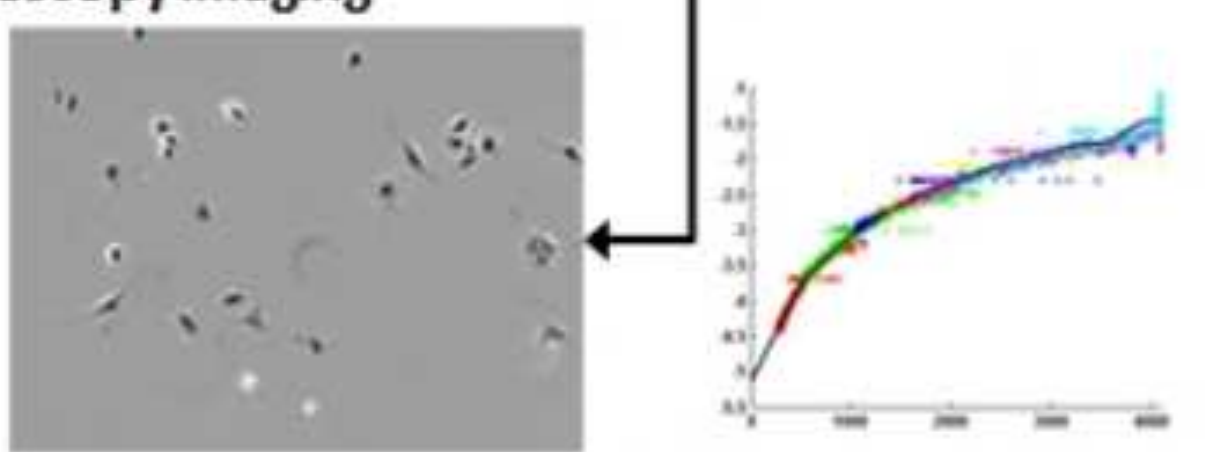

Restored irradiance $E$

Estimate cell-sensitive camera response function 